\title{
RESEÑA
}

\section{HISTORIA DE LA DIVISIÓN DE INVESTIGACIONES FUCS DIEZ AÑOS}

\author{
Omar Segura MD ${ }^{1,2}$, Merideidy Plazas ${ }^{1}$, Licet Villamizar ${ }^{1}$, Luidy Geovana González ${ }^{3}$, William Rojas MD \\ Esperanza Peña ${ }^{1}$, Guillermo Sánchez MD ${ }^{1}$, Edgar Muñoz MD
}

\section{Resumen}

Este artículo presenta una historia comparada de las fases de desarrollo que ha tenido la División de Investigaciones de la FUCS, desde su embrión en 1997, su origen oficial en 2002, y el proceso de crecimiento y consolidación desde entonces en aspectos tales como objetivos, personal, docencia, hasta llegar al Grupo de Investigación y División que es hoy en día. Para el efecto se utilizó un enfoque cualitativo que combinó los abordajes de entrevista en profundidad, análisis documental y revisión de archivos fotográficos, seguido de organización del material de referencia y compilación en una versión manuscrita para socialización y acuerdo entre coautores. El proceso histórico resultante muestra a la División de Investigaciones, al cumplir sus primeros diez años, como una estructura administrativa y académica universitaria orgánica de la Vicerrectoría Académica en plena actividad, dinámica, cuyos miembros entienden tanto su potencial como sus propios límites de actuación, y enfrentan cada día nuevos desafíos en lo tocante a pesquisa, investigación e innovación en salud.

Palabras clave: historia, investigación cualitativa, entrevistas como asunto, fotografía, recursos humanos en salud, epidemiología, salud pública.

\section{HISTORY OF FUCS RESEARCH DIVISION: 10TH ANNIVERSARY}

\begin{abstract}
This compared review describes the stages of development of the FUCS Research Division, from its "embryo" in 1997, its official birth in 2002, and its growth and consolidation process, since that time, in aspects such as objectives, staff, instruction, up to the creation of today's Research Group and Division. A qualitative approach was used for in-depth interviews, documental analysis and photography files review, followed by, organizing reference data and compiling data in a written version in order to socialize and achieve an agreement among co-authors. Accordingly, this review pictures the Research Division on its 10th anniversary as an administrative and organic university academic structure, part of the Office of Academic Affairs, as a full dynamic activity, who's members understand its potential as well as its own limitations to act, with daily new challenges as to inquiries, research and innovation in health issues.

Key words: history, qualitative research, issue interviews, photography, human resources for healthcare, epidemiology, public health
\end{abstract}

Fecha recibido: julio 18 de 2012 - Fecha aceptado: agosto 17 de 2012

I División de Investigaciones, Fundación Universitaria de Ciencias de la Salud, FUCS, Bogotá DC, Colombia.

Doctorado en Salud Pública, Universidad Nacional de Colombia.
3 División de Aseguramiento de la Calidad, FUCS.

4 Hospital de San José.

5 Vicerrector Académico, FUCS. 


\section{Introducción}

Investigar en ciencias de salud es ante todo un proceso y un método hacia un nuevo, mayor y mejor conocimiento del paciente como individuo, y del balance salud/enfermedad para el colectivo. Es ante todo -sobre todo al interior de un hospital universitario- una actividad muy humana que transcurre en el tiempo, que involucra el quehacer de profesionales, técnicos y personal administrativo, cada uno y en conjunto con una serie de aportes y desarrollos (o retrocesos) que inciden en la evolución de una unidad o de una institución y que con el paso del tiempo se ha vuelto compleja al involucrar otras ciencias y disciplinas para resolver preguntas que cada profesión de salud ya no puede solventar actuando por separado. Esta es una opinión compartida por varios investigadores: Jara ${ }^{1}$, por ejemplo, comenta que aunque las ciencias y procesos de investigación en salud tienen un notable desarrollo, han ocurrido dentro de los cánones o paradigmas de las ciencias clásicas, cuando podrían obtener mayor beneficio y profundidad en sus investigaciones si asumen una aproximación multi/transdisciplinar.

Cadena, en su prólogo sobre el itinerario histórico de la Sociedad de Cirugía de Bogotá ${ }^{2}$, menciona cómo diversos gestores, en una serie concatenada de acontecimientos, preceden a la ocurrencia de aquellos sucesos que marcan el devenir de las instituciones. O también, de aquellas estructuras al interior de las instituciones, las cuales juegan un rol preponderante en el desarrollo de las mismas, hechos que con el paso del tiempo, al ser sopesados en forma primero cronológica, después comparativa y luego analítica, se convierten en eso que conocemos como historia. La historia, según Delgado ${ }^{3}$, al ser elemento básico en la cultura de toda persona-cualquiera sea su formación y nivel de estudios, y el sector salud no es la excepción-, se torna imprescindible para la formación de la manera de pensar, de actuar y de ser de todos los sujetos. Por su parte, Bloch -citado por Álvarez Scanniello - o $\mathrm{Kocka}^{5}$, teorizaron acerca de la necesidad de contar con elementos históricos para luego trascenderlos a un nivel que permita examinar hechos y procesos históricos que podrían ser pasados por alto, si no se tuvieran en cuenta realidades parecidas, incluso en el mismo lugar, pero ubicadas en diferentes contextos.

Así, la evolución de una institución o de una de sus áreas, puede ser analizada según esta perspectiva en particular: el abordaje de la historia de la Fundación Universitaria de Ciencias de Salud (FUCS) como un todo, de la División de Investigaciones como una parte específica, se pueden enmarcar en esta forma de pensar. El punto de partida es considerar que, en su momento, sus dirigentes fueron conscientes de la necesidad de contar con un equipo multidisciplinario que brindara el apoyo y solventara las necesidades investigativas en salud dentro de la institución, tomando así la decisión de crear la División. Ahora, sea una perspectiva histórica o una organizacional, es desaconsejable que un miembro de una comunidad o institución ignore cómo esta se originó, cual fue su evolución en forma general -o mejor, a través de etapas de desarrollo-, cuál es su presente y para dónde apunta su futuro, ya que en no pocas ocasiones su destino individual y profesional puede estar ligado al comunitario e institucional.

Por consiguiente, esta compilación -aunque brevetiene por objetivo dar cuenta y poner en perspectiva los hechos más importantes detrás de la formación de la División de Investigaciones, orgánica de la Vicerrectoría Académica, al cumplir sus primeros diez años de operación en julio de 2012.

\section{Métodos}

Para escribir este manuscrito, se realizaron a lo largo del primer semestre de 2012, actividades de indagación/pesquisa, aplicando un enfoque cualitativo con un abordaje de carácter documental y biográfico ${ }^{6-9}$, desarrollando como actividades: a) entrevistas en profundidad a personas que habían sido directoras de la División (Tabla 1), y consultas con aquellas quienes, en el primer trimestre de 2012, todavía trabajaban o de alguna forma estaban en conectadas con la División; b) consulta a documentos tales como acuerdos del Consejo Superior, actas del Comité de Investigaciones (que, como se verá, fue instrumental para el surgimiento de la División) y diversos manuales, en 
particular aquellos que hacen parte de la documentación administrativa creada para la División entre 2002 y 2003; c) recopilación y análisis de fotografías y otros documentos, en especial en formato electrónico; d) organización del material y redacción del presente texto; y e) socialización y acuerdo con los diferentes testigos y participantes de esta compilación, para así escribir el documento en su versión definitiva.

\section{Resultados}

\section{La División de Investigaciones es pensada: 1997-2002}

Los orígenes de la División de Investigaciones se remontan a la creación del cargo de Coordinador de Investigaciones y del Comité de Investigaciones de la FUCS, en julio y agosto de 1997 respectivamente, cuyo objetivo primario era el de promover, asesorar y coordinar todo lo relacionado con investigaciones en la Fundación, contando con participación de los jefes de departamento, los decanos de todas las facultades, el coordinador del Comité y una asesora metodológica ${ }^{1}$ de posgrado.

La División de Investigaciones y el actual Comité de Ética en Investigación con Seres Humanos (CEISH) de la FUCS comparten orígenes comunes. En efecto, la primera reunión de un "Comité de Ética en Investigaciones” ocurrió el 1998-05-22, habiendo asistido William Rojas (endocrinólogo), acompañado de Claudia Medina (nutricionista), Mauricio Ruiz (nefrólogo) Humberto Reynales (internista y epidemiólogo), y Sergio Parra (ginecobstetra, rector de la FUCS en 2012). En la reunión, el doctor Rojas explicó las funciones del Comité: a) prestar apoyo inicial a los proyectos de investigación generados al interior de la Facultad de Medicina, a nivel de pregrado o posgrado; b) dar aval a las investigaciones realizadas en el Hospital, en particular aquellas que contaran con el apoyo de la industria farmacéutica; y c) hacer el seguimiento a los proyectos de los residentes, dando así mismo paz y salvos para graduación. El doctor Rojas también expuso cuáles deberían ser los requisitos

I En las Facultades de Enfermería o de Instrumentación FUCS, las asesorias para el pregrado se llevaban a cabo en la respectiva cátedra de investigación. y deberes del Comité desde la ética en investigaciones, incluyendo la conformación del acervo documental basado en las recomendaciones del Comité Internacional de Armonización sobre Investigaciones en Salud.

En julio de 1998, se creó el Comité General de Investigaciones (CGI), con el doctor Rojas como coordinador y la decana de enfermería, Ana Julia Carrillo, como secretaria del mismo. Este comité, constituido por los decanos o sus representantes, tenía por objetivo trazar las políticas generales de investigaciones de la Fundación. En 1999, el CGI recomendó el desarrollo de líneas de investigación interconectadas en cada facultad de la Fundación; la Facultad de Enfermería crearía al año siguiente la línea de Cuidado en Enfermería y Salud Comunitaria.

En palabras del propio doctor Rojas, antes de 2000, "la investigación generada en la FUCS se realizaba de manera independiente, difusa, hasta que se realizaron los primeros esfuerzos por estandarizar la actividad". Así mismo, las actas recogidas dan muestra de un proceso aún informal, que podía sesionar oficialmente o dedicarse a responder inquietudes de los residentes, según hubiera quórum o no. Su temporalidad primero fue irregular, luego quincenal -más o menos-, pero con los primeros esbozos de una de las actividades de la futura División: la asesoría y crítica metodológica de los proyectos, que incluía la presentación de los proyectos por los residentes de la respectiva especialidad, para que personal del comité ofreciese lineamientos y sugerencias al diseño de los estudios y al análisis estadístico, y la sustentación del (ante)proyecto, acompañada por sus respectivos profesores, complementada con la generación de observaciones y sugerencias para ajustar los trabajos por parte de ambos grupos.El archivo de los proyectos se realizaba entonces en discos magnéticos (floppy diskettes); los diseños de los estudios solían ser de carácter descriptivo en su mayor parte y uno que otro analítico o experimental.

Hacia comienzos del año 2000, ya estaba instituida la reunión con los residentes a punto de graduarse, para informarles los requisitos finales antes de la entrega del trabajo de grado, y estaba ya constituida una jornada dedicada a la presentación por los residentes de las 
investigaciones realizadas. Está registrada en las actas digitales la solicitud de unos residentes de anestesiología "para que a los residentes se les de tiempo en sus rotaciones para poder recibir apoyo de investigaciones ya que muchas veces las actividades asistenciales les impiden cumplir con los cronogramas y reuniones del Comité de Investigaciones. " Hecho aparentemente trivial pero que tendría impacto en la futura conformación y quehacer de la División. En abril de este año el CGI organizó un curso de introducción a la investigación, abierto para docentes de todas las facultades, en el que participaron veinte personas.

El proceso de autoevaluación de la Facultad de Medicina, que comenzó en junio de 2001, llevó al equipo de trabajo a considerar la existencia de líneas de investigación definidas; después, el coordinador del comité preparó un documento denominado "Plan de Consolidación de la División de Investigaciones", creado en forma digital en 2001-09-03. En dicho documento, el doctor Rojas consignó el reconocimiento nacional e internacional de los profesionales del Hospital de San José y el desarrollo de la investigación de las aulas de la FUCS, en cuanto "nuevo brazo académico de la Sociedad de Cirugía de Bogotâ". Comentó el doctor Rojas la perspectiva planteada por la Ley 30 de 1992, y escribió que “...existiendo desde hace tres años la División de Investigaciones de la Fundación, es oportuno darle cuerpo y estructura administrativa a dicha sección para fortalecer la investigación en nuestra institución."

Fue en ese momento que se determinó la conformación de un equipo de trabajo para la integración física, administrativa y operacional de las tareas de ética en investigación, asesoría metodológica y docencia en investigación, para estar listo en un máximo de 18 meses. Esta estructura tendría como misión construir un camino que permitiera generar cultura investigativa dentro de la comunidad universitaria, y estaría conformada por un director científico, un coordinador de la sección de bioestadística y metodología, un coordinador de epidemiología y una secretaria; para ese momento, la FUCS asignó un espacio en la antigua área de consulta externa del Hospital de San José y empezaron a delimitarse las funciones de la futura División. En mayo de 2002, el doctor Rojas escribió: "La investigación es la columna vertebral sobre la cual reposa la solidez científica de una institución universitaria, es la forma en que una institución aporta conocimiento para la mejoría de la sociedad en términos de salud, bienestar, tecnología, educación y desarrollo. Por tal motivo la Fundación Universitaria de Ciencias de la Salud ha tenido dentro de su estructura administrativa y dentro de su fundamentación académica el núcleo de investigaciones, el cual se encuentra claramente descrito en el Proyecto Educativo Institucional, en los estatutos de la Fundación y en el estatuto docente.(...) la investigación (es) un proceso continuo y permanente que la comunidad académica realiza, con el objeto de identificar realidades, causas, efectos, tendencias, así como de socializar sus hallazgos, para que sean un fundamento de transformación de las actividades educativas y de formación institucionales."

En este momento, la División fue planteada no sólo como una oficina operativa --con los cargos y las funciones generales antes mencionados-, sino como un ente asesor del Rector para establecer áreas y líneas en las facultades, con autonomía presupuestal, capaz de manejar una convocatoria en pregrado y posgrado y de aglutinar la capacitación y seguimiento de los docentes y los especialistas en entrenamiento. El doctor Rojas aventuró un lapso de tiempo de dos a cuatro años para tener consolidada su propuesta de División, conformada como una Unidad de Investigación Clínica (un futuro e independiente "Centro de Investigaciones en Salud") capaz de contratar con laboratorios la realización de sus estudios, que contara incluso con un bioterio para suplir algunas investigaciones de especialidades como ortopedia y cirugía plástica, y formar su propio personal al nivel de maestría para que se enfocara en las líneas y áreas de énfasis en investigación del momento.

\section{La División de Investigaciones es conformada: 2002-2003}

Para 2002, la División de Investigaciones se conforma en un contexto interno de desinterés de los docentes por la investigación, y externo asociado con la visita 
del Ministerio de Educación Nacional para la obtención del registro calificado². Por Acuerdo del Consejo Superior, el cargo de Director de la División de Investigación fue creado en 2002-06-22, y la médica epidemióloga Martha Beatriz Montenegro fue nombrada como Directora: desde aquí comenzó el trabajo correspondiente a su organización y consolidación.

Entre las primeras tareas se contaron la creación de las políticas de funcionamiento de la División, la creación de los comités de investigación en las diferentes facultades; se ideó y gestionó el apoyo económico de los proyectos de investigación -que sería la base de la Convocatoria Interna, buscar personal idóneo para apoyar la parte académica en las facultades. El informe de gestión julio-noviembre de 2002 dio cuenta de varios proyectos programados: establecimiento de un diagnóstico o línea de base y levantamiento de un plan estratégico y un análisis de debilidades, oportunidades, fortalezas y amenazas (DOFA) que llegó a proyectar incluso un centro de investigación en ciencias básicas; la conformación del cuerpo docente; el curso académico para los residentes y el proyecto de capacitación docente a nivel de diplomado y un encuentro de trabajo programado para noviembre de ese año. Cabe destacar elementos del DOFA, por cuanto representaban las esperanzas y los problemas de la División en ese entonces: apoyo de las directivas, programas de posgrado, revista dedicada, áreas de práctica, convenios interinstitucionales y vinculación de docentes por perfil, en un entorno al que le faltaba construir una cultura clara de investigación reflejada en líneas, tiempo protegido, incentivos, infra y superestructura dedicadas y específicas.

Se dio apertura aproximadamente en septiembre de 2002 a las asesorías a los residentes con apoyo de los doctores Ricardo Londoño (ortopedista del Hospital de San José) y Mónica Sossa (coordinadora del comité de infecciones del Hospital de San José); los trabajos derivados de los estudios asesorados serían fuente de una primera jornada de investigaciones en enero de 2003, aunque su comienzo oficial ocurrió en 2005. El grupo de docentes lo conformaban, además, Henry Oli-

2 Contemplado en la ley 749 de 2002, reglamentado luego por el Ministerio de Educación Nacional. veros (médico, intensivista y epidemiólogo), Juliana Velosa (odontóloga, epidemióloga clínica), Socorro Moreno (psicóloga, epidemióloga clínica), Álvaro Sanabria (cirujano de cabeza y cuello) y Francy Licet Villamizar (instrumentadora quirúrgica). La Directora de la División era, al mismo tiempo, su representante ante la Facultad de Medicina. En esta época, había un coordinador de investigaciones para cada facultad de la FUCS; este docente de la División se encargaba de asesorar todos los trabajos de investigación de la facultad, de impartir docencia en pregrado y posgrado, y de diversas tareas administrativas relacionadas.

Entre el 06 y el 11 de noviembre del mismo año se realizó un evento de investigación en el municipio de Villa de Leyva, cuyo objetivo primario era completar el proceso conformación de la División. En esta reunión se analizó la misión, la visión y la estructura inicial de una cátedra de investigaciones unificada para los programas de pregrado (Foto 1).

\section{La División de Investigaciones se consolida: 2003-2007}

A partir de julio de 2003, bajo la conducción de la segunda directora de la División, la médica epidemióloga clínica Marcela Gómez (Foto 2), se levantó un diagnóstico actual de cada facultad y programa ${ }^{3}$, conformándose a continuación un proyecto estratégico para el período 2003-2005. Los elementos considerados en ese momento fueron la investigación formativa aplicada, las líneas de investigación, la asignación de recursos y la formación de investigadores. Desde lo administrativo, se creó el primer Manual de Funciones, luego un Procedimiento de Inducción, y se inició el Catálogo de Investigaciones y Publicaciones; vendría posteriormente lo que el informe de gestión 2003 denominó "la construcción de una cultura en investigación y la conformación de semilleros de investigación, quienes serán los grupos de investigación del futuro".

En ese momento, los profesores ya daban asesorías metodológicas para los diferentes profesionales de la

3 En este momento, los programas en pregrado eran medicina, enfermería, citohistotecnología e instrumentación quirúrgica; en posgrado medicina y enfermería. 


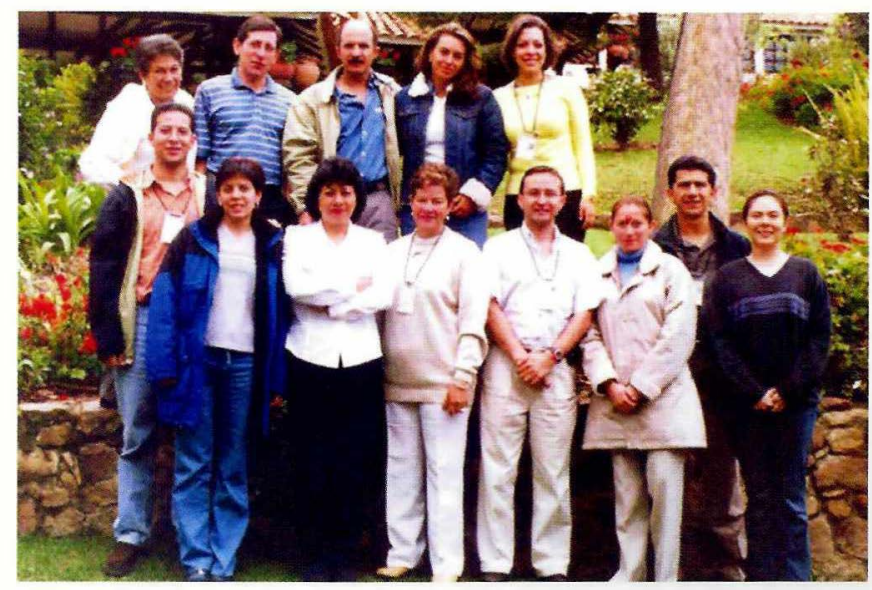

Foto I. Personal de la División de Investigaciones en el encuentro de Villa de Leyva, noviembre de 2002. De izquierda a derecha, primera fila: Álvaro Sanabria, Juliana Velosa, Rosa Coral, Julialba Alarcón, Edgar Acuña, Licet Villamizar, Sergio Parra y Socorro Moreno. Segunda fila: Amparo Benavides, Henry Oliveros, Ricardo Londoño, Margarita Ruiz y Martha Montenegro (Directora de la División)

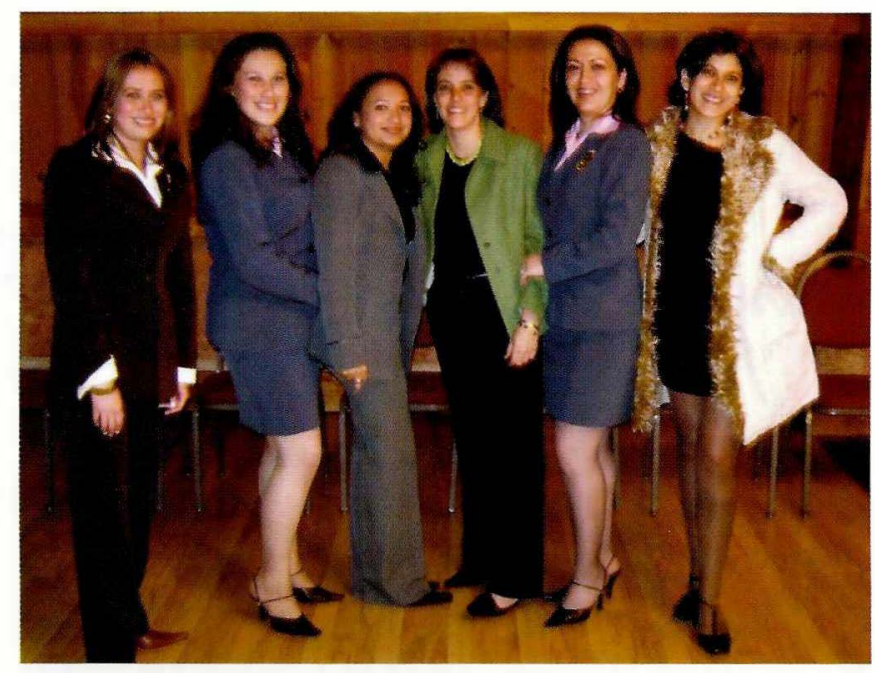

Foto 2. Mujeres en la División de Investigaciones, 2003-2006. De izquierda a derecha: Licet Villamizar, Geovana González, Merideidy Plazas, Marcela Gómez (Directora de la División), Alexandra Prieto (Secretaria Aseguramiento de la Calidad) y Erika Vargas.

FUCS y del Hospital, a un ritmo de 24 horas semanales; el enfoque inicial fue hacia las facultades de citohistotecnología e instrumentación. En ese mismo año comenzó un proyecto de monitorías de investigación, bajo un concepto de teoría económica que encontró aplicación en la pedagogía, el de learning by doing (aprender haciendo). Se dictaron cursos de investigación biomédica, bioestadística y paquetes estadísticos, tales como el Statistical Package for $t$ Social Sciences (Paquete Estadístico paa Ciencias Sociales), más conocido como SPSS. Se programó el primero de los encuentros de investigación. El grupo de profesores estaba conformado por Carlos DíazGranados (infectólogo, epidemiólogo), Ilse Vásquez (médica epidemióloga), Licet Villamizar (instrumentadora, epidemióloga), Merideidy Plazas (bacterióloga, epidemióloga), Juanita Paz (neumóloga, epidemióloga) y Erika María Vargas (estadística, especialista en análisis demográfico).

En 2004, en el contexto nacional, el Consejo Nacional de Acreditación inició los procesos de acreditación universitaria. La FUCS definió entonces el PEI (Plan Educativo Institucional), en el cual quedaron plasmadas las orientaciones generales de lo que las directivas y el cuerpo académico definieran que serían las políticas de investigación, con lo cual la División hizo una definición de objetivos, revisión y ajustes con la creación de nuevos documentos. Entre tanto, ocurrió un hecho trascendental para la División: se creó la Vicerrectoría Académica, de la cual la División sería orgánica posteriormente; existía ya un Comité Primario (encargado de recursos financieros, presupuesto) para medicina, y un Comité Curricular conformado por las cuatro decanaturas. De este tiempo datan los primeros acercamientos a Colciencias a través de Marcela Espinel; por entonces, los proyectos de posgrado eran concebidos y funcionaban desde los estudiantes, mientras que en pregrado las opciones de trabajo de investigación se enfocaron hacia desarrollo, pasantías, asistencia o monografías, con una tendencia hacia los estudios económicos y la búsqueda de literatura científica.

En ese momento, todos los residentes debían graduarse con un trabajo de investigación, tras haber recibido al menos clases de metodología de investigación en dos semestres, a mitad de carrera; los trabajos contaban ya con la asesoría metodológica de la División, pero al no contar con asesor temático, la responsabilidad de su desarrollo, conducción y publicación corría a cargo de los estudiantes. La sustentación de los trabajos se realizaba frente a jurados de la División, pero sin un formato estructurado para tal fin. Todavía no 
se realizaba el Examen de Calidad de Educación Superior (ECAES) ${ }^{4}$. En el segundo semestre de 2004 comenzaron las clases que se conocieron luego como los módulos de investigación: como todo proceso al comienzo, hubo caos y resistencia entre los docentes. Diferente al énfasis actual, no se abordó por entonces el tema de lectura crítica.

Para 2005, en respuesta, la organización de la División hizo cambios internos y se crearon los cargos de Coordinador de Pregrado y de Posgrado (vigente hasta la fecha para medicina y para enfermería). Se crearon las asignaturas comunes de investigación básica, investigación media, medicina basada en evidencia, enfermería basada en evidencia y métodos cualitativos en investigación. Empezaron a realizarse los exámenes ECAES para médicos y enfermeras, frente a lo cual la Facultad de Medicina requirió apoyo de la División en la preparación de preguntas y sesiones de información y alistamiento.

En el período 2005-2006 se reactivaron los grupos de investigación, empezando por el Grupo División de Investigaciones $\mathrm{FUCS}^{5}$; de este tiempo data el primer intento de formación de una red colaborativa y fue creada la Oficina de Planeación que manejaba inicialmente trece procedimientos operativos estándar (POE), un manual de procedimientos, y un procedimiento de validación. En esta época se integraron al grupo los profesores Juan Camilo Aponte (médico, epidemiólogo clínico), Andrés Espinosa-Bode (médico, epidemiólogo clínico) y Jesús Reyes (estadístico). La FUCS creó un programa de rotación especial de investigación para internos interesados en el tema; el primero de ellos fue el médico Carlos Alberto Castro, quien se integraría al cuerpo docente de la División dos años después. Las tareas de la División en relación con los exámenes ECAES incluyeron ahora las facultades de enfermería e instrumentación quirúrgica.

4 Con la Ley 1324 de 2009, se hizo obligatorio presentar este examen para optar al título profesional respectivo; desde este acto administrativo cambió su nombre por el de SABER PRO.

5 Grupo reconocido en categoría D hasta 2011, número de registro COL0033032,http://20I.234.78.173:8080/gruplac/jsp/visualiza/visualizagr. jsp?nro $=00000000005170$.

\section{La División de Investigaciones crece: 2007- 2010}

En 2007, la División -bajo el mando de Esperanza Peña, que había ingresado como docente el año anterior- renovó el personal (Foto 3) para darle un carácter más diverso -multidisciplinar, si se quiere-, y reformó la estructura de los programas en metodología de investigación, incluyendo elementos de capacitación por competencias y desempeños y diferentes referencias bibliográficas.

En palabras de la magister Peña: "Siempre sentí mucho apoyo de las directivas, incluso para las cosas técnicas: las personas que vinieron detrás de mí enfrentaron una situación más difícil, con pocos recursos [la conformación de la División]; yo seguí el camino de mi antecesora [Marcela Gómez]; y me sentí orgullosa al decir que la FUCS era la única universidad que de verdad se preocupaba por la investigación. Considero que la investigación debe ser vinculante con otros procesos; debe ser metódica, constructiva, sin atajos, y debe partir desde la propia universidad, desde sus propias preguntas."

En 2008, el requisito para los estudiantes de último año en -particular en pregrado- de entregar un trabajo

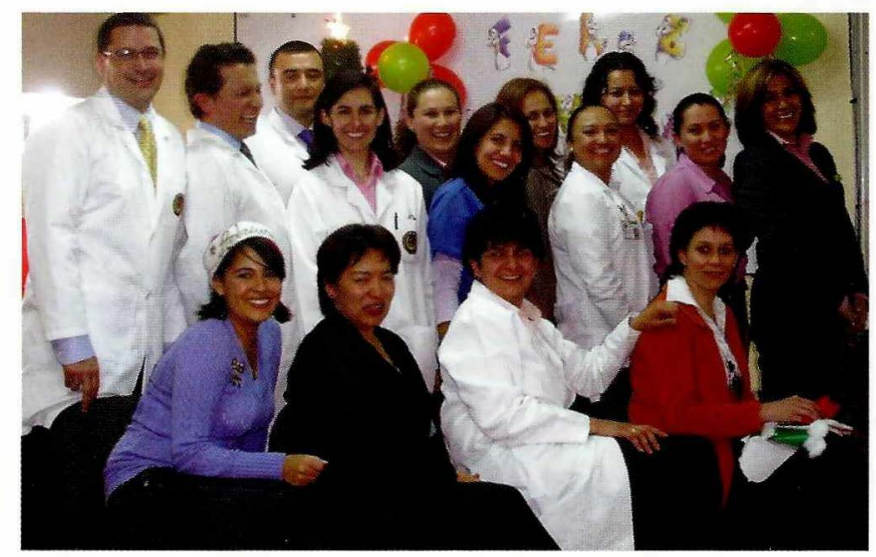

Foto 3. Personal de la División de Investigaciones, 2007-2010. De izquierda a derecha, sentadas:Adriana Buitrago, María Nelcy Rodriguez, Esperanza Peña (Directora de la División) y Paula Prieto. De pie: Alejandro Colmenares, Cesar Piñeros, Alfredo Portilla, Magda Alba, Geovana González, Erika Vargas, Martha Arévalo, Merideidy Plazas, Ingrid Arévalo, Licet Villamizar y Patricia Pérez (secretaria, Comité de Ética en Investigación con Seres Humanos, CEISH). 
de grado incluyó nuevas posibilidades: preparar un trabajo de investigación, realizar una pasantía en investigación, redactar una monografía o crear material digital en formato de $\mathrm{CD}$. Un aspecto importante de su proceso cambió con la transferencia de la responsabilidad por esos trabajos de los residentes a los docentes. Para esta época, todos los trabajos ya contaban con asesoría metodológica -cuya naturaleza al final de cuentas orientaba hacia la investigación formativa, de carácter observacional-; sin embargo, esta asesoría era realizada con el docente, en un proceso secuencial que lo llevara desde la formulación de una propuesta, pasando por la preparación del protocolo, hasta la redacción y publicación del respectivo artículo; la sustentación, por su parte, se realizaba en la respectiva facultad y los estudiantes eran evaluados por el respectivo especialista docente y por el asesor metodológico, y el trabajo era calificado por jurados. Ocurrió un cambio en el pensum, para que todas las asignaturas de la facultad de medicina buscaran desarrollar competencias investigativas en los residentes de cada especialidad.

La División incorporó a su portafolio de servicios la realización del curso de Buenas Prácticas Clínicas a partir de 2009, en respuesta a las directrices emanadas del Invima a propósito del cumplimiento de requisitos relacionados con la buena marcha de los ensayos clínicos y fue instrumental en la conformación de un grupo de investigación: el Centro de Investigación en Medicina Crítica y Aguda (CIMCA) ${ }^{6}$ del Hospital Universitario Infantil de San José, bajo el mando de los doctores César Enciso y Mario Gómez Duque.

\section{La División de Investigaciones encara el futuro: 2010-2012 (y más allá...)}

Hasta 2010, los planes estratégicos habían sido formulados de manera específica y por cada facultad de la FUCS, siendo bianuales hasta 2010; desde 2011, lo fueron a cuatro años, en consonancia con el Proyecto Educativo Institucional.

A raíz de una serie de recomendaciones realizadas por el Consejo Nacional de Acreditación durante la visita

6 Grupo reconocido por Colciencias en la convocatoria 20II, número de registro COL009663I, http://201.234.78.173:8080/gruplac/jsp/visualiza/ visualizagr.jsp?nro $=000000000097 \mid 4$. de pares de 2009, a comienzos de 2010 fue creada la figura del "Gestor de Conocimiento", para la generación y desarrollo de proyectos. El cargo lo ocupó el médico y biólogo molecular Carlos Hugo Escobar, quien también asumió en interinidad la dirección de la División entre julio y diciembre de aquél año; su gestión -dada la situación- se orientó a mantener lo ganado y avanzado?.

En enero de 2011 llegó a la dirección el médico epidemiólogo Guillermo Sánchez Vanegas, candidato a doctorado en salud pública en ese momento. Tras un análisis del recorrido histórico y del entorno alrededor de la División, el nuevo Director planteó transformaciones estructurales de cara a la consolidación del cuerpo docente, simultáneo con el componente administrativo. Hasta este momento su énfasis era acompañar la realización de los trabajos de investigación por los estudiantes de posgrado; pese a la importancia de esta labor, el nuevo Director planteó reformular el enfoque hacia una transformación de la cultura existente, para que se basara en el concepto de grupos de investigación, con líderes investigadores docentes, que articularan en sus proyectos a los estudiantes de posgrado y pregrado, dando lugar a un escenario en el cual se integraran la investigación aplicada y la formativa.

Gracias a la modificación del reglamento de posgrado y a la aprobación por el Consejo Superior en agosto del 2011, del documento Análisis, actualización y proyección de las políticas de investigación en la Fundación Universitaria de Ciencias de la Salud, se reafirmó la voluntad de las directivas de la universidad para ofrecer un apoyo frontal y sostenido a la investigación. Este apoyo llevó más lejos las acciones de la Vicerrectoría Académica, que a comienzos del 2011 reestructuró el Comité Institucional de Investigaciones, el cual opera como ente rector de la investigación en la FUCS. Cabe anotar que este comité es presidido por el Vicerrector Académico y cuenta con la presencia del Rector, el Director de la

7 Es en esta época, julio de 2010, cuando el primer autor se incorporó a la División como Profesor Asistente, habiendo trabajado desde junio de 2004 con la facultad de enfermería de la FUCS, en calidad de instructor de epidemiología y de gerencia de proyectos para el posgrado de gerencia en salud. 
División y de los delegados de todas las facultades de la universidad.

Todos estos cambios contribuyeron a la expansión de los grupos de investigación en la universidad, pasando de 8 en el 2010 a 29 en el 2012, logrando el reconocimiento por Colciencias de 17 de ellos. La División, a su vez, reorganizó su propio grupo de investigaciones, generándose tres líneas de investigaciones: a) epidemiología clínica, b) investigación en pedagogía, y c) garantía de calidad y gestión hospitalaria. Concomitante con este crecimiento, la naturaleza de la convocatoria interna para financiación de proyectos de investigación cambió del carácter trimestral y puntual original a una estructura permanente, primero, y de tipo redistributivo de los recursos, después, con el fin de poder ofrecer subvención a un mayor número de proyectos. También se formularon dos nuevas convocatorias para fortalecer los semilleros de investigación ${ }^{8}$ y la movilidad de los investigadores.

8 Cuya conformación oficial ocurrió mediante acuerdo del Consejo Superior número 2080 de 2010.
Bajo esta nueva política, también la universidad ha fortalecido sus procesos de seguimiento administrativo a los proyectos de investigación que financia, en un esfuerzo por profesionalizar el ejercicio de la investigación entre nuestros docentes, al tiempo que se optimiza al máximo el uso de los recursos, generando la mayor productividad posible.

A comienzos de 2012 la División ha enfrentado el aumento de la demanda de los cursos de metodología de investigación y las asesorías metodológicas en dos sentidos: uno, diseñando y dando inicio al diplomado en epidemiología clínica, de carácter semestral, con la participación de diez profesores (Foto 4) y residentes de la FUCS; otro, con la incorporación de dos nuevos miembros en la división, los médicos y epidemiólogos clínicos Lizet Paola Moreno y Jimmy Arévalo Romero. Es previsible que este curso de acción evolucione en la génesis de un curso especializado de mayor alcance -especialización o maestría- en epidemiología clínica o metodología de investigación.

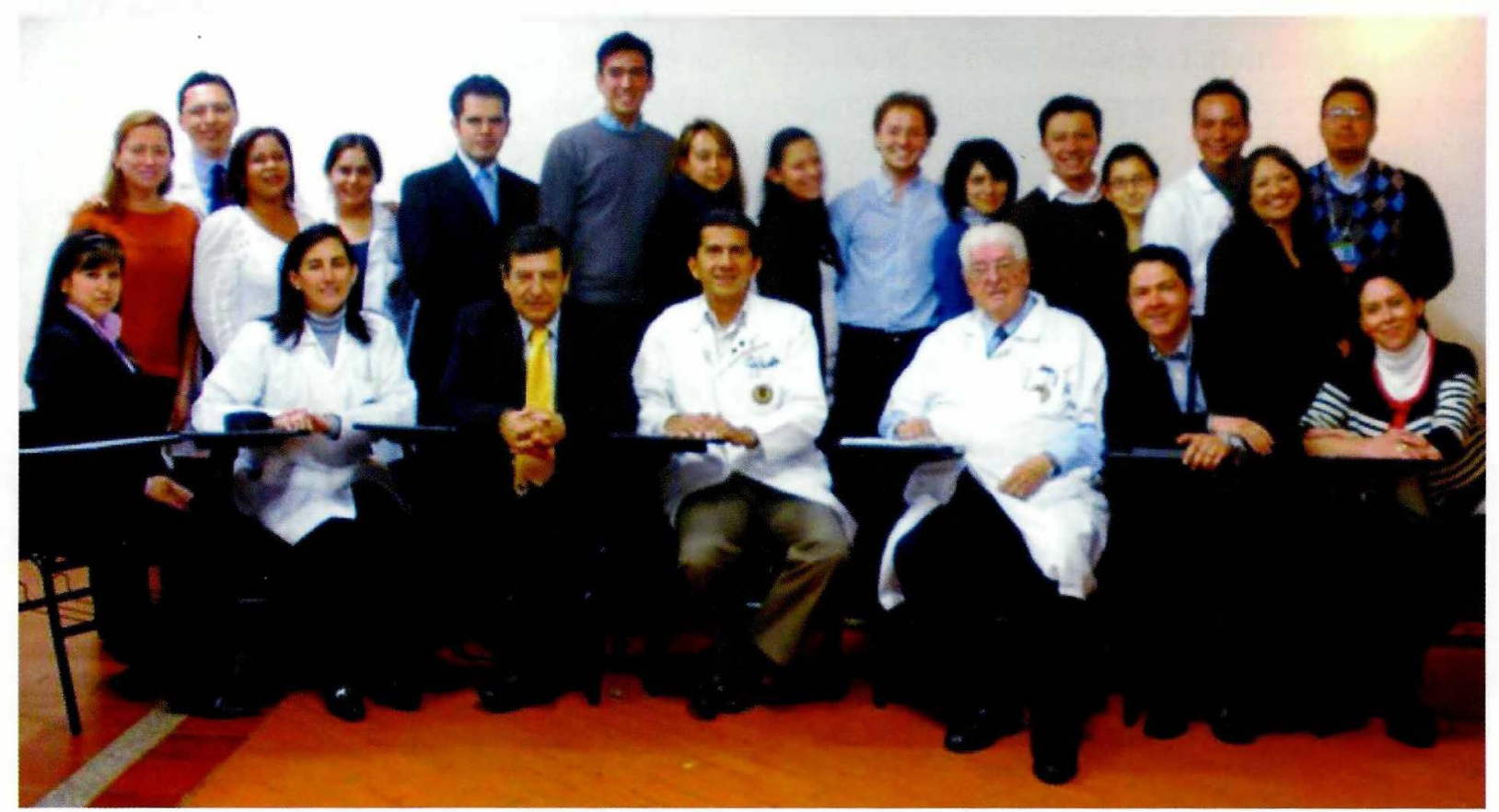

Foto 4. Personal de la División de Investigaciones, julio de 2012. De izquierda a derecha, sentados: Ángela Báez, Magda Alba, Edgar Muñoz (Vicerrector Académico), Sergio Parra (Rector), Roberto Jaramillo (Rector Honorario), Guillermo Sánchez (Director de la División) y Paula Prieto. De pie: Licet Villamizar, Carlos Castro, Celmira Laza, Lizet Paola Moreno, Oscar Gamboa, Jimmy Arévalo, Nataly Preciado, Diana Buitrago, Pedro Montoya, María Angélica Rodríguez, César Piñeros, Alexandra Castiblanco, Carlos Hugo Escobar, Merideidy Plazas y Omar Segura. 


\begin{tabular}{|l|l|l|l|}
\hline \multicolumn{4}{|c|}{ Tabla I. Directores de la División de Investigaciones FUCS, 2002-2012 } \\
\hline Nombre & \multicolumn{1}{|c|}{ Profesión } & \multicolumn{1}{|c|}{ Posgrados } & \multicolumn{1}{c|}{ Período } \\
\hline Martha Beatriz Montenegro & Médica & Epidemiología & junio 2002 - noviembre 2003 \\
\hline Marcela Gómez Suárez & Médica & Epidemiología & noviembre 2003 - febrero 2007 \\
\hline Esperanza Peña Torres & Enfermera & $\begin{array}{l}\text { Administración } \\
\text { Epidemiología }\end{array}$ & marzo 2007- julio 2010 \\
\hline Carlos Hugo Escobar & Médico & Biología Molecular & encargado: julio 2010 - diciembre 2010 \\
\hline Guillermo Sánchez Vanegas & Médico & $\begin{array}{l}\text { Epidemiología } \\
\text { Salud Pública }\end{array}$ & enero 201I - actual \\
\hline
\end{tabular}

\section{Discusión}

La División de Investigaciones pasó de tener cuatro miembros en 2002 -profesionales- a tener dieciseis en 2012 - un candidato a PhD, dos estudiantes de doctorado, maestros y especialistas, rurales e internos- ; de una oficina pasó a contar con tres y un salón de clase asignado; de no tener grupos de investigación en 2002 pasó a tener su propio grupo de investigación con tres líneas y a apoyar en el reconocimiento de 17 grupos de investigación hacia 2012; de ideas y aportes sobre la docencia en investigación en 2002 pasó a tener a la fecha toda una estructura académica-docente, operacional, siempre en periódica evaluación; de carecer de recursos asignados en 2002 pasó a coordinar una convocatoria interna para trabajos de investigación de carácter permanente hacia 2012; de una estructura unidisciplinar-epidemiología clínica- y una estructura operativa reactiva (causa-efecto) en 2002, pasó en 2012 a tener un enfoque multi/transdisciplinar, proactivo, ejecutivo, en camino de hacerse, si se quiere, todavía más "complejo" y más capaz de asumir proyectos de largo alcance y duración en el quehacer de la Salud Pública y en el saber de sus distintas disciplinas y especialidades.

La División de Investigaciones es, pues, una estructura orgánica de la Vicerrectoría Académica en pleno crecimiento, dinámica, consciente de sus capacidades como de sus propios límites de actuación. Ahora, para todo proceso científico y administrativo surgen inquietudes, se avizoran desafíos, y se trazan planes: ¿Qué quiere hacer la División de Investigaciones, en el horizonte de una reforma académica universitaria que será objeto de debate este año? ¿Cuáles son los objetivos y metas de la División hasta 2015, fecha final de vigencia del Plan Educativo Institucional actual? ¿Qué puede ser capaz de lograr la División, sus objetivos y metas, dada la evolución aquí presentada, y qué espera lograr? ¿Qué enseñanzas deja para la comunidad FUCS, para otras instituciones, el devenir histórico de la División de Investigaciones de la Fundación Universitaria de Ciencias de la Salud? Las respuestas quedan en manos de sus directivas, de los miembros de la División, y de la propia comunidad académica universitaria que la alberga.

\section{Referencias}

1. Jara MI. La investigación en salud pública: una reflexión epistemológica desde las ciencias de la complejidad. Rev Gerenc Polit Salud 2009;8:32-56.

2. Cadena D. A manera de prólogo. In: Sociedad de Cirugía de Bogotá - Itinerario histórico desde el 22 de julio de 1902. Bogotá: La Sociedad; 1998:9-11.

3. Delgado G. Conceptos y metodología de la investigación histórica. Rev Cub Salud Pub 2010;36:9-18.

4. Scanniello JÁ. Sobre el método comparado. Bol Hist Econ (Montevideo) 2008;VI:18.

5. Kocka J. Comparison and beyond. History and Theory 2003;42:39-44.

6. Antonio Bolívar JD. Biographical-narrative Research in Iberoamerica: Areas of Development and the Current Situation. Forum: Qualitative Social Research $2006 ; 7$.

7. Cornejo M. El Enfoque Biográfico: Trayectorias, Desarrollos Teóricos y Perspectivas. Psykhe 2006;15:95-106.

8. Díaz G. El enfoque cualitativo de investigación en la salud pública contemporánea. Rev Cub Salud Pub 2010;36:30-7.

9. Sanz A. El método biográfico en investigación social: Potencialidades y limitaciones de las fuentes orales y los documentos personales. Asclepio 2005 LVII:99-115 